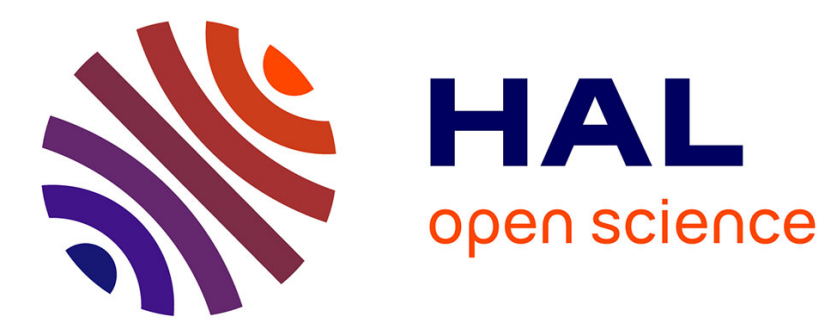

\title{
Les grandes surfaces alimentaires contre le territoire... tout contre
}

\author{
Michaël Pouzenc
}

\section{To cite this version:}

Michaël Pouzenc. Les grandes surfaces alimentaires contre le territoire... tout contre. Pour : enquêtes et témoignages, 2012, 215-216, pp.255 - 261. 10.3917/pour.215.0255 . hal-01568946

\section{HAL Id: hal-01568946 \\ https://hal.science/hal-01568946}

Submitted on 26 Jul 2017

HAL is a multi-disciplinary open access archive for the deposit and dissemination of scientific research documents, whether they are published or not. The documents may come from teaching and research institutions in France or abroad, or from public or private research centers.
L'archive ouverte pluridisciplinaire HAL, est destinée au dépôt et à la diffusion de documents scientifiques de niveau recherche, publiés ou non, émanant des établissements d'enseignement et de recherche français ou étrangers, des laboratoires publics ou privés. 


\title{
LES GRANDES SURFACES ALIMENTAIRES CONTRE LE TERRITOIRE... TOUT CONTRE
}

\author{
Michaël Pouzenc
}

GREP | «Pour »

2012/3 N²15-216 | pages 255 à 261

ISSN 0245-9442

Article disponible en ligne à l'adresse :

http://www.cairn.info/revue-pour-2012-3-page-255.htm

\section{Pour citer cet article :}

Michaël Pouzenc, « Les grandes surfaces alimentaires contre le territoire... tout contre », Pour 2012/3 (N²15-216), p. 255-261.

DOI $10.3917 /$ pour.215.0255

Distribution électronique Cairn.info pour GREP.

(C) GREP. Tous droits réservés pour tous pays.

La reproduction ou représentation de cet article, notamment par photocopie, n'est autorisée que dans les limites des conditions générales d'utilisation du site ou, le cas échéant, des conditions générales de la licence souscrite par votre établissement. Toute autre reproduction ou représentation, en tout ou partie, sous quelque forme et de quelque manière que ce soit, est interdite sauf accord préalable et écrit de l'éditeur, en dehors des cas prévus par la législation en vigueur en France. Il est précisé que son stockage dans une base de données est également interdit. 


\section{Michaël POUZENC}

Géographe, Université de Toulouse,

UMR Dynamiques rurales

\section{Les grandes surfaces alimen- taires contre le territoire... tout contre}

es deux tiers des achats alimentaires s'effectuent en grande surface, de manière très stable durant toutes les années 2000 (67,1\% en 1999 et $67,3 \%$ en 2006) (INSEE, 2007). Actrice essentielle de l'approvisionnement alimentaire des ménages, la grande distribution est pourtant régulièrement décriée, entre autres pour son rôle dans la standardisation de l'alimentation, l'appauvrissement des cultures alimentaires et leur déconnexion vis-à-vis des territoires de production. En effet, son déploiement vise à capter des flux de consommateurs, auxquels est proposée une offre commerciale de plus en plus homogène, au fur et à mesure que disparaissent les petits commerces alimentaires indépendants et que sont reproduits partout les mêmes concepts de supermarchés avec les mêmes assortiments. Le rôle uniformisant de la grande distribution alimentaire tient aussi fortement à sa participation à la normalisation et à l'intensification des systèmes logistiques et des filières de production agroalimentaire. La centralisation toujours plus poussée des achats, effectués par un nombre toujours plus réduit de grands groupes de distribution, tend à favoriser l'appro- 
visionnement auprès d'un nombre limité de grands groupes et PME de l'agroalimentaire plutôt qu'auprès d'une myriade de petits producteurs. Ainsi, la mutation du commerce accompagne largement la concentration des entreprises agroalimentaires et des exploitations agricoles. L'évolution des caractéristiques des produits est tout aussi remarquable, la grande distribution nécessitant la fabrication massive de produits homogènes, conditionnés pour bien supporter un transport en grandes quantités sur de grandes distances, et dotés d'une durée de conservation maximale. Les modes de fabrication de ces produits s'en trouvent eux-mêmes profondément transformés. Enfin, la mutation du commerce exerce une forte influence sur les modes de consommation, faisant évoluer l'origine et la part qu'occupe chaque produit dans le panier de la ménagère. Au total, la grande distribution participe largement à la massification et à la mondialisation des approvisionnements.

Les grandes et moyennes surfaces apparaissent donc avant tout comme des machines à homogénéiser l'espace. Plus précisément, elles sont au cœur d'un fonctionnement pluri-scalaire hiérarchisé : l'internationalisation des distributeurs est forte mais s'est révélée plus laborieuse qu'escompté ; elle laisse aujourd'hui encore un rôle majeur d'organisation aux groupes de distribution à l'échelle nationale, pour laquelle des organisations régionales jouent un rôle de relais en matière de logistique et de coordination des magasins affiliés, de même que les organisations locales lles points de vente eux-mêmes) jouent un rôle de relais pour identifier les opportunités locales de développement, adapter l'offre de produits et la communication.

Ce système a-t-il pour seul effet de distendre le lien entre alimentation et territoire ? Sous la prédominance de l'échelle nationale, les quelques marges de manœuvre laissées aux échelles régionale et locale ne sont-elle pas porteuses de relations plus complexes ? Différentes études de cas en Midi-Pyrénées nous ont permis de relever comment la grande distribution alimentaire s'adapte aux territoires ruraux qu'elle investit, voire valorise certaines formes de ruralité.

\section{S'adapter au contexte}

Dès ses débuts, la grande distribution a fait preuve de capacités d'adaptation aux contextes régionaux et locaux, que ce soit par les choix d'implantation de ses points de vente, l'ajustement certes marginal des gammes de produits proposés aux habitudes locales de consommation, ou encore certaines formes d'intégration au milieu local.

Ainsi, l'historique des créations de supermarchés et d'hypermarchés en MidiPyrénées permet de montrer selon quelles logiques le tissu des grandes surfaces alimentaires s'est progressivement mis en place (M. Pouzenc, 1999, 2010). Au fil des décennies, les grandes surfaces alimentaires ont connu un essor considérable et ont 
peu à peu couvert l'ensemble de la région, en prenant position tout d'abord dans les principaux pôles urbains. Le tissu des grandes surfaces, dans sa configuration de la fin des années 1970, paraît ainsi quasiment identique au tissu des agglomérations de plus de 5000 habitants. Au début de la décennie 1980, une rupture importante apparaît dans cette expansion. Les créations de supermarchés s'opèrent tous azimuts, non seulement dans les communes urbaines, comme durant les décennies précédentes, mais également dans des bourgs dépassant parfois à peine les 500 habitants, ou encore dans des communes rurales situées en périphérie des petites villes. La décennie 1980 correspond ainsi très nettement à la phase de conquête du milieu rural par les distributeurs, parachevée dans les années 1990 à un rythme plus modéré. Durant la décennie 2000 , le quadrillage des espaces ruraux par les grandes surfaces alimentaires a continué de se renforcer partout où s'annonçait une croissance démographique, qu'il s'agisse des espaces ruraux éloignés des villes ou, plus fortement encore, de ceux en voie de périurbanisation. L'implantation des supermarchés a même devancé l'actualisation par l'INSEE de ses délimitations des couronnes périurbaines et une étude plus fine permettrait de faire ressortir une adaptation de l'appareil commercial à différents types d'espaces périurbains, par exemple selon leur éloignement plus ou moins grand de l'agglomération et des grands axes de circulation.

L'adaptation au contexte régional et local ne se limite pas aux implantations de points de vente. L'offre de produits définie à l'échelle nationale demande à être partiellement ajustée aux spécificités régionales, car à ce niveau les habitudes alimentaires ne sont pas totalement homogénéisées. L'adaptation de l'offre s'accompagne d'une adaptation de la politique de communication. L'étude d'un corpus de prospectus publicitaires (M. Pouzenc, 1999) nous avait permis de relever que les références à l'espace ou au territoire ne sont pas toutes valides à la même échelle. Autant faire mention de Dijon, pour la moutarde, semble pouvoir fonctionner auprès des consommateurs de la France entière, autant la référence à Millau, pour la charcuterie, ne doit pas être très évocatrice au-delà des régions Midi-Pyrénées et LanguedocRoussillon. Aux références d'envergure nationale viennent ainsi s'ajouter quelques références à résonance plus régionale, telles que le jambon de Lacaune ou le cassoulet de Castelnaudary. Cette adaptation aux spécificités régionales est limitée mais tenace : les prospectus des années 2010 ne démentent pas ceux des décennies précédentes. En outre, la promotion des produits régionaux s'est particulièrement développée durant les années 1990, avec le succès des marques de distributeurs dédiées aux produits de terroir. Qu'il s'agisse, par exemple, de Reflets de France chez Carrefour ou de Nos régions ont du talent chez E. Leclerc, ces marques ombrelles rassemblent une série de produits alimentaires dont l'emballage magnifie le lieu de production, son patrimoine et ses savoir-faire ancestraux; bon nombre de ces produits comportent de surcroît 
un signe officiel de la qualité et de l'origine, tel que l'AOC (Appellation d'origine contrôlée).

De la même manière, l'effort d'intégration des supermarchés au milieu local, particulièrement mis en évidence en espace rural avec l'étude de cas sur la Barousse (M. Pouzenc, 1999), continue de se vérifier à l'heure actuelle. Dans le contexte particulier d'une petite vallée de moyenne montagne à la population majoritairement âgée, et pour se différencier de la concurrence des petites villes avoisinantes, la direction du supermarché de Loures-Barousse continue de miser sur les relations d'interconnaissance qui se cultivent sur son point de vente, aussi bien entre clients qu'entre clients et employés, habitants eux aussi des villages voisins. Le recrutement local du personnel, ainsi que son faible taux de renouvellement, constituent un pointclé de cette intégration au milieu local. Celle-ci s'opère également à l'extérieur du magasin, non seulement car le directeur et le personnel ont noué des liens d'amitié avec un grand nombre d'habitants, mais aussi car ils sont impliqués dans les milieux associatifs. Le fait que le directeur du supermarché ait passé son permis de chasse et qu'il ait rejoint l'association des chasseurs apparaît comme un élément d'intégration particulièrement significatif, compte tenu de l'importance accordée à la chasse en Barousse. L'intégration au milieu local passe enfin par la mise en avant d'une vingtaine de produits locaux (fromages et autres produits laitiers, salaisons, volailles, fruits et légumes, biscuits, miel...). Même s'ils représentent une part minime du chiffre d'affaires, ils sont perçus comme importants pour l'image du magasin.

Aussi puissante soit-elle, la grande distribution alimentaire n'est donc pas en mesure d'appliquer en toute indépendance la même politique standardisée à tous les espaces qu'elle investit. En espace rural, dans un contexte concurrentiel difficile lié notamment à une faible densité de la clientèle et à une forte évasion commerciale vers les pôles urbains, sa réussite passe au contraire par son intégration au milieu local et la prise en compte de ses spécificités. En ce sens, l'essor de la grande distribution ne se résume pas à une normalisation des espaces qu'elle investit ; les espaces ruraux connaissent un renouvellement de leur signification spécifiquement rurale car se reproduisent des contextes d'action qui leur sont propres. Cela n'est pas démenti pas les évolutions récentes.

\section{Cultiver la différence}

La décennie 2000 se différencie des précédentes par le fait que la croissance des grandes surfaces n'implique plus la disparition massive des petits commerces. Entre 2000 et 2006, le nombre d'alimentations générales et supérettes a ainsi diminué de $2 \%$, tandis que les commerces alimentaires spécialisés ont diminué de $5 \%$ (INSEE Enquête annuelle entreprise dans le commerce, 2000 et 2006). Mais le petit com- 
merce en voie de stabilisation dans les années 2000 est très différent de celui qui a décliné pendant trente ans. Par exemple, si la raréfaction des boucheries et des boulangeries traditionnelles se poursuit lentement, elle est en bonne partie compensée par le développement des « commerces alimentaires spécialisés divers », vocable sous lequel l'INSEE inventorie de nouvelles catégories d'établissements, dédiés par exemple aux plats cuisinés, aux produits bio ou aux produits du commerce équitable.

À ces évolutions s'ajoute un engouement pour les relations de proximité entre producteurs et consommateurs. Les initiatives en la matière sont foisonnantes (M. Pouzenc et al., 2008). Elles sont tout à fait minimes en termes de chiffres d'affaires, comparativement aux grandes surfaces, mais elles suffisent à montrer que l'environnement de la grande distribution a changé. La construction d'une proximité avec le client, à la fois spatiale, sociale et culturelle, est devenue un enjeu majeur de ces dernières années.

Or la grande distribution intègre rapidement, lorsqu'elles peuvent contribuer à son succès, ces initiatives affichées initialement comme alternatives (M. Pouzenc et M. Guibert, 2010). Ainsi note-t-on, tout d'abord, le regain d'intérêt des distributeurs pour les réseaux sous franchise de petits commerces de proximité, dont les résultats sont honorables, dans ces années 2000 où le modèle de l'hypermarché donne des signes d'essoufflement. Le plus important réseau d'alimentations générales et de supérettes est celui du groupe Casino, avec près de 4000 établissements aussi bien en zone urbaine qu'en zone rurale, dont 1200 magasins à l'enseigne Vival, plutôt dédiée aux espaces ruraux (www.casino-proximite.fr, consulté en août 2008). Le groupe Carrefour, depuis sa fusion avec Promodès, dispose d'environ 2500 magasins de moins de $400 \mathrm{~m}^{2}$, dont 1500 sous l'enseigne Proxi, implantée principalement en milieu rural. Ce groupe souligne également, dans une campagne publicitaire concernant son enseigne Shopi (Le Monde, 18 juin 2008, p. 7), l'importance des petits supermarchés pour les habitants de l'espace rural. Cette publicité laisse toutefois perplexe par sa référence au village de Mauliac... inventé de toutes pièces.

En outre, dans une société de consommation et de développement durable soucieuse d'équité, la grande distribution est amenée à déployer des stratégies d'affichage d'un certain respect des producteurs. À la promotion des produits de terroir des années 1990 s'ajoutent, dans les années 2000, la promotion du commerce équitable, affichant le respect des petits producteurs du Sud, tout comme la promotion des produits bio et de la consommation locale, à renfort de grandes affiches clamant des contrats justes noués par des distributeurs avec des agriculteurs qui produisent propre et près de chez nous (par exemple Les Alliances locales d'E. Leclerc en pleine page du journal Le Monde, 26 novembre 2010 ou en séries de vidéos sur YouTube en 2011 et 2012). Ces différentes 
démarches, peu ou prou sous la bannière du développement durable, participent ainsi à la reformulation d'une certaine « demande de ruralité ».

Bien entendu, l'adaptation à la grande distribution d'initiatives conçues au départ comme alternatives présente des limites. La grande distribution repose sur une centralisation des achats et une massification des flux de marchandises; ses démarches pour instaurer une proximité entre consommateurs, terroirs et producteurs, si appréciée dans d'autres formes de vente, en demeurent forcément réduites. L'instrumentalisation des terroirs pour l'animation des grandes surfaces laisse parfois perplexe, qu'il s'agisse du verrat en plastique grandeur nature, auprès d'un étal de saucissons, ou du fermier à l'accent rocailleux, à demi métamorphosé en présentateur de jeux télévisés. Lors de ses campagnes publicitaires, si Carrefour affirme qu' « être utile chaque jour, c'est s'engager auprès des PME et des producteurs locaux », l'échelon local dont il est question correspond... au pays dans son entier (« acheter français en France et brésilien au Brésil ») (Le Monde, 20 juin 2008, p. 17). La commercialisation massive en grande distribution interroge également les agriculteurs bio, dont les modes de production, dans certains cas, font largement appel à des opérations manuelles; le passage à des modes de production industriels sans compromission avec les principes de départ de l'agriculture biologique paraît purement et simplement inconcevable à une partie d'entre eux. Des débats similaires traversent les milieux du commerce équitable.

Par ailleurs, la grande distribution représente certes une formidable opportunité pour les territoires dont la production était jusque-là confidentielle et qui, lorsqu'elle est référencée par un distributeur, voient s'accroître de manière considérable les volumes écoulés et voient assurée leur vitalité économique. Les territoires dans ce cas sont nombreux, tels les terroirs africains du thé Rooibos, les terroirs andins du quinoa ou les terroirs viticoles australiens et les terroirs fromagers français. En même temps, ces territoires peuvent se trouver dans une situation fragile, car rien ne garantit que leur investissement massif dans telle ou telle production sera durable. Que les modes alimentaires changent, que la grande distribution se tourne vers d'autres fournisseurs, et s'ouvre une crise de reconversion. Un exemple précis peut illustrer cette instabilité : dans le plus important hypermarché de l'agglomération toulousaine, entre 2001 et 2006, le renouvellement des fromages de chèvre en rayon libre service est très important. Seuls 19 produits déjà présents en 2001 l'étaient toujours en 2006 ; 38 autres, soit les deux tiers, avaient disparu, pendant que 26 produits qui n'existaient pas en 2001 apparaissent sur le linéaire en 2006. Selon les cas, l'explication de ces changements peut varier, qu'il s'agisse d'un renouvellement des fournisseurs auxquels s'adresse Carrefour, d'une restructuration des entreprises de ce secteur, par des rachats ou des fusions de sociétés, ou encore de nouvelles stratégies de grands groupes agroalimentaires souhaitant investir le 
créneau fromage de chèvre où ils étaient absents jusque-là ( $M$. Pouzenc et J. Pilleboue, 2006).

En conclusion, la grande distribution connaît le même jeu d'opportunités et de contraintes que bien d'autres acteurs économiques. Devant arbitrer en permanence entre la standardisation nécessaire aux économies d'échelle et la spécification nécessaire à une pénétration plus fine des marchés, elle applique les mêmes modèles dans tous les espaces qu'elle investit, en même temps qu'elle développe une forme de territorialisation : tout en étant actrice du changement dans les espaces ruraux, la grande distribution prend en compte leurs spécificités et ce faisant, participe à leur reproduction en tant que contextes d'actions spécifiques.

Plus encore, les grandes et moyennes surfaces ne se contentent pas de s'adapter au contexte ; elles participent à la construction de la ruralité qui leur paraît commercialement valorisable. Car une constante demeure au fil des temps : tout l'art du commerce, qu'il soit grand ou petit, consiste à cultiver la différence. Ainsi, nul doute que la grande distribution saurait s'adapter à une culture alimentaire qui s'ancrerait plus fortement dans les territoires, pour peu que les consommateurs aient davantage conscience de ces ancrages, aient pris goût à les inscrire dans leur vie quotidienne et aient appris à les réclamer. Une question d'éducation?

\section{À lire}

INSEE, « Les petites entreprises du commerce depuis 30 ans », Insee Première n 831, 2002. www.insee.fr/fr/ffc/docs_ffc/ip831.pdf

INSEE, Le Commerce en France - édition 2007, 2007. http://www.insee.fr/fr/publications-etservices/sommaire.asp?id=167\& nivgeo $=0$

Michaël Pouzenc, Grande distribution alimentaire et recomposition des territoires. Étude de stratégies d'acteurs dans des zones rurales de Midi-Pyrénées, Thèse de doctorat en Études rurales mention Géographie, Université de Toulouse-Le Mirail, 1999, 2 volumes.

Michaël Pouzenc et Jean Pilleboue, « Le terroir dans les rayons des hypermarchés. Le fromage de chèvre », Midi-Pyrénées Patrimoine n 8, 2006, p. 7-21.

Michaël Pouzenc et al., Les relations de proximité agriculteurs-consommateurs : Points de vente collectifs et AMAP en Midi-Pyrénées, Rapport d'étude, Toulouse, 2008. http://dynamiquesrurales.univ-tlse2.fr/accueil/productions/rapports-de-recherche/.

Michaël Pouzenc et Martine Guibert, «Produits et filières : entre le rentable et l'équitable », dans Philippe Dugot et Michaël Pouzenc (dir.), Territoires du commerce et développement durable, éd. L'Harmattan, coll. «Itinéraires géographiques », 2010, p. 191-236. 